\title{
An Effective Research on Data Mining Techniques for Intrusion Detection \& Learning Classes
}

\author{
Venkateswara Rao Ch, G. Siva Nageswara Rao
}

\begin{abstract}
It exhibits that the accumulation based alignment is altogether introduced mentioned than normal unmarried-model strategies; supervised acquirements beats unsupervised learning, and accretion the amount of apocryphal negatives connects to university precision. It demonstrates activity over consequences facts. For alignment facts, this cardboard proposes and checks an unmonitored, association primarily based acquirements including that keeps up a organized chat advertence of addled successions observed. All through able abstracts surges of great breadth to analyze inconsistencies. In unsupervised learning, burden based techniques are activated to archetypal basal conduct groupings. This outcomes in a classifier announcement a ample accession in alignment attention for abstracts streams absolute cabal blackmail irregularities. This accouterments of classifiers allows the unsupervised manner to accord with exhausted established changeless acquirements methodologies and lifts the functionality over supervised acquirements methods. One of the bottlenecks to frame backpack chat advertence is adaptability. For this, a executed adjustment is proposed and done utilizing Hadoop and Map lessen machine.We could augment the plan CRISP-DM archetypal in the accompanying means To activate with, we will assemble an actual framework to bolt applicant addition as beck utilizing apache abysm and abundance it on the Hadoop conveyed certificate framework (HDFS) and afterwards that administer our methodologies. Next, we will administer Map Reduce to amount adapt abolish amid examples for a specific client's adjustment assumption data.
\end{abstract}

Keywords : classes, learning, models, GBAD, Threat, supervised, LIBSVM, CRISP-DM model.

\section{INTRODUCTION}

As new models location unit made and past ones refreshed to be greater genuine, the littlest sum right fashions location unit disposed of to constantly maintain up accomplice troupe of explicitly okay cutting-edge fashions. An non-compulsory manner to cope with supervised acquirements is unsupervised gaining knowledge of, which can be electively affiliated to sincerely untagged information-i.e., abstracts amidst which no focuses location assemblage simply acclaimed as aberrant or nonpeculiar. Graph primarily based abnormality vicinity (GBAD) is one fundamental array of unsupervised acquirements (cook dinner and Holder, 2007; Eberle and Holder, 2007; cook dinner and Holder, 2000), in any case, has verifiably been restricted to static, restricted length datasets. This restricts its application to streams related with business official threats that will in general have boundless length and threat designs that advance after some time.
Applying GBAD to the business official threat issue in this way needs the models utilized be accommodating and efficient. Including these characteristics empower effective models to be built from colossal measures of developing data. In this treatise, we watch out for strong business official threat acceptance as a beck mining disadvantage and able aspect 2 systems (supervised and unsupervised learning) for efficiently sleuthing irregularities in beck abstracts (Parveen, McDaniel et al., 2013). To administer abstraction development, our supervised methodology keeps up partner advancing outfit of different OCSVM models (Parveen, Weger et al., 2011). Our unsupervised methodology joins severa GBAD models in the partner outfit of classifiers (Parveen, Evans et al., 2011). The outfit trade technique is expected in the two instances to remain the collection present day seeing that the circulate develops. This herbal procedure capability complements the classifier's survival of concept drift due to the fact the conduct of every real and unwell-conceived operators fluctuates after a while.

From accomplished decades on lath quick beforehand central the web congenital up data, new appeal ranges for $\mathrm{PC}$ web accept risen. At the akin period, far extensive alter beforehand central the LAN and WAN ask for ranges in the organization, business, industry, allowance and amusing allowance capacity fabricated us added abased on the PC systems. these ask for levels fabricated the net a associate agreeable consciousness for the corruption and giant acknowledgment for the community. An arresting plan to do or an endeavor to accede movement for acquaintance humans came to be a abhorrent dream for the others. In unnumerable event, awful-natured accomplishments fabricated this terrible dream to acknowledgment to ea reality.

Notwithstanding hacking, new elements like worms, Trojans, and infections gave additional frenzy into the organized society. As the present situation could be a tolerably new improvement, web deadly implements feeble. However, because of the acknowledgment of PC net, their property and be that as it may, producing reliance on them, information of the hazard will have despoiling outcomes. Protecting such a critical foundation has come back to be the need one investigation length for unnumerable scientists.on

Revised Manuscript Received on September 10, 2019.

Dr. Venkateswara Rao*, Computer Science and engineering, Siddhartha Institute of Engineering \& Technology, Ibrahimpatnam, Hyderabad,T.S., India.

G. Siva Nageswara Rao, Department of Computer Science\& engineering,Koneru Lakshmaiah Education Foundation, Vaddeswaram, Guntur,A.P.,India. 
this mission, basically middle across the exam of the existing direction in Intrusion Detection arrangements (IDS) and to take a gander at a conservative gift difficulty that maintains amid this have a look at space. In similarity to a minimized expand and nicely - stayed examine stages, IDS will be dynamic earth of examination. However, because of its obligation vital nature, it is allured noteworthy consideration with admire to itself. The thickness of examination regarding this amount is consistently ascent and consistently added specialists are assemblage afraid amidst this apple of work. The crisis of a barter beachcomber of cyber or net animosities isn't just a befalling that should be trusted, still will advance whenever. The present pattern for the IDS is saved from a solid securing course of action, in spite of the fact that the fundamental accepted.

If there should be an occurrence of partner interruption attempt, the game plan is in a situation to note and to report it. at the point when the recognition is solid, the resulting pace ought to be to monitor the on the web (reaction). Furthermore, the IDS game plan will be moved up to a partner Intrusion Detection and Reply Arrangement (IDRS). However, no fragment of the IDS is by and by at a totally dependable dimension. Indeed, even by and by, scientists are unit in the meantime associated with working every identification and answer groups of the framework. A primary blow inside the IDS is that the guarantee for the interruption location This is the reason why in various cases IDS utilize the interruption discovery. Amid this procedure, IDS is truly helping the online assurance ace and it isn't sufficiently legitimate to make sure in its own. The motive is the powerlessness of IDS guides of movement to look the brand new or adjusted attack designs. However assuming the contemporary method for identity methods has advanced the popularity rate. Anyways, there is an all-inclusive philosophy to move.

There are 2 primary manners by which for distinguishing interruptions, signature-primarily based and inconsistency based interruption popularity. Inside the early approach, attack plots or the sports of the interloper is modeled (assault mark is modeled). Right here the sport plan can motion the interruption when a in shape is prominent. However, inside the ensuing neither strategy, ordinary activities of the web are modeled. Amid this technique, the sport plan can enhance the alert whilst the demonstration of the online doesn't coordinate on board its customary conduct. There's a further Intrusion Detection (identification) strategy that is uproarious particular based totally interruption recognition. Amid this approach, conventional deeds (expected conduct) of the host is counted and after sculpturesque. Oversee well worth for safety, opportunity of approach for the host is constrained. in this paper, those approaches can be quickly mentioned and notion about.

The accepted of having partner participant getting to the game plan coming up short on being able to take note of it's the most noticeably bad dream for each net insurance officer. As the present ID data isn't right masses to upgrade a solid identification, heuristic approaches can be an exit plan. With respect to the last line of insurance, thus as to cut the quantity of inconspicuous interruptions, heuristic ways like Honey Jars (HP) might be conveyed. Nectar containers might be introduced on each course of action and go about as deceive or distraction for an asset.

Another fundamental issue amid this study range is that the speed of discovery. PC networks have a dynamic nature in a genuine sense that information and learning inside them are persistently evolving. Accordingly, seeing partner interruption precisely and properly, the course of action must include constant. working in the genuine length most effective to play out the location inside the true period, yet is to change to the brand new additives within the framework. true duration working IDS is relate geared up exam range admired by incalculable analysts. The more part of the examination works are unit intended to acclimate the first sum robust philosophies. The point is to make the asked for strategies affordable for the real period utilization.

From a divergent viewpoint, 2 manners by which might be conceived in asking for IDS. In this connection, IDS can be whichever have built up or net based for the most part. In the host set up IDS, requesting will just guard its very own inborn machine (its host). On the advantageous hand, inside the net set up IDS, the ID system is some way or another circulated on board the system. Along those traces, although the operator installation gaining knowledge of is extensively requested for, a circulated recreation plan will comfortable the internet all in all. in this shape, IDS can manage or display web firewalls, web switches or internet switches simply because the purchaser machines.

make rectification in the final paper but after the final submission to the journal, rectification is not possible. In the formatted paper, volume no/ issue no will be in the right top corner of the paper. In the case of failure, the papers will be declined from the database of journal and publishing house. It is noted that: 1 . Each author profile along with photo (min 100 word) has been included in the final paper. 2. Final paper is prepared as per journal the template. 3. Contents of the paper are fine and satisfactory. Author (s) can make rectification in the final paper but after the final submission to the journal, rectification is not possible.

\section{LITERATURE SURVEY}

S. Duque and Omar [2] proposed a K-Mean bunching on NSL-KDD dataset. "The count is associated on different five gatherings. The best results are procured when 22 clusters were used. In like manner, K-Mean gathering is used as a piece of blend approaches", like B. Sharma and H. Gupta [3] uses two frameworks alliance run and gathering. "Apriori and K-Mean are used to perceive the interferences. The test is done on KDD'99 dataset. The cpu Utilization $(74 \%)$, usage measures are execution time $(120 \mathrm{~ms})$ and memory use (54\%)".

Ravale and Nilesh et al. [4] projected the ewer approach of K-Mean and RBF portion limit of SVM. "The preciseness ultimate outcome of the ewer approach is ninety three and therefore the characteristic proof rate is ninety five. wherever Chao and sebaceous cyst et al. [5] projected a 
hybrid methodology of K-Mean and K-NN. The accuracy result's higher for instance ninety nine during this work. each hybrid methodologies use KDD'99 dataset".

Liang and Nannan et al. [7] proposed a system "which mixes of K-Mean and Fluffy C Mean (FCM) figurings to discard false positive from the dataset DARPA 2000. The completion of the work is the effect of FCM figuring is better than whatever of K-Mean gathering". Zhengjie and Yongzhong [8] proposed to approach the "K-Mean and particle Swarm Advancement system (PSO-KM). The acknowledgment rate of realized ambushes is $75.82 \%$ and of cloud, strikes is $60.8 \% "$ ". "To upgrade the execution of SVM, Horng and Yang, et al. [9] one and a half SVM with different leveled batching. The BRICH dynamic bundling figuring is used for expansion decision framework to discard unimportant features from a dataset with the objective that SVM describes the data even more exactly. The accuracy is $95.72 \%$ and the false positive rate is $0.7 \% "$.

\section{RELATED WORK}

Anchoring PC and system data is critical for associations and people in light of the fact that traded off data can cause extensive harm. To dodge such conditions, interruption recognition frameworks are vital. As of late, extraordinary machine learning approaches have been proposed to enhance the execution of interruption identification frameworks. Wang et al. [1] proposed an interruption discovery structure dependent on SVM and approved their strategy on the NSL KDD dataset. They guaranteed that their technique, which has $99.92 \%$ viability rate, was better than different methodologies; nonetheless, they didn't make reference to utilized dataset measurements, number of preparing, and testing tests. Moreover, the SVM execution diminishes when huge data are included, and it's anything but a perfect decision for examining colossal system traf c for interruption location. Kuang et al. [2] connected a mixture model of SVM and KPCA with GA to interruption location, and their framework demonstrated a $96 \%$ recognition rate. They utilized the KDD CUP99 dataset for the check of their framework, yet this dataset is described by restrictions. One model is repetition be one-sided to all the more much of the time happening records. They connected KPCA for highlight decrease, and it is restricted by the likelihood of missing imperative highlights on account of choosing top rates of the vital segment from the main space. Likewise, the SVM isn't fitting for substantial data, for example, observing the high transfer speed of the system.

Interruption location frameworks give help with identifying, forestalling, and opposing unapproved get to. Accordingly, Aburomman and Reaz [3] proposed an outfit more tasteful technique, which is a blend of PSO and SVM; this more tasteful beat different methodologies with $92.90 \%$ exactness. They utilized the learning revelation and data mining 1999 (KDD99) dataset, which has the recently referenced downsides. Moreover, the SVM is certifiably not a decent decision for immense data investigations, since its execution corrupts as data estimate increments.

Raman et al. [4] proposed an interruption recognition component dependent on hypergraph hereditary calculation (HG-GA) for parameter setting and highlight determination in SVM. They asserted that their technique beat the current methodologies with a $97.14 \%$ identification rate on a NSL KDD dataset; it has been utilized for experimentation and approval of interruption recognition frameworks.

The security of system frameworks is a basic issues in our day by day lives, and interruption location frameworks are signicant as prime protection techniques. In this manner, Teng et al. [5] led critical work. They built up their model dependent on choice trees (DTs) and SVMs, and they tried their model on a KDD CUP 1999 dataset. The outcomes demonstrated an exactness achieving $89.02 \%$. Notwithstanding, SVMs are not favored for overwhelming datasets on account of the high calculation cost and poor execution.

Farnaaz and Jabbar [6] built up a model for an interruption discovery framework dependent on RF. They tried the viability of their model on a NSL KDD dataset, and their outcomes exhibited a $99.67 \%$ recognition rate contrasted and J48. The fundamental confinement of the RF calculation is that numerous trees may make the calculation moderate for continuous forecast. Elbasiony et al. [7] proposed a model of interruption recognition dependent on RF and weighted k-implies; they approved their model over the KDD99 dataset. The framework exhibited outcomes with $98.3 \%$ precision. The RF isn't reasonable for anticipating genuine traf $\mathrm{c}$ on account of its gradualness, which is because of the arrangement of an expansive number of trees. Furthermore, the KDD99 dataset demonstrates couple of restrictions as previously mentioned.

\section{EXISTING WORK \& RESULTS}

It serves the point of giving a great deal of detail on explicitly anyway every method touches base at location business official threats and the manner in which outfit models region unit planned, adjusted and disposed of. The primary portion goes over supervised learning completely and in this manner the second fragment is going over unsupervised studying. each consists of the recipes essential to understand the inner operations of each class of gaining knowledge of learning.

\section{A. Supervised Learning}

In a lump, a model is made abuse one classification bolster vector machine (OCSVM) (Manevitz and Yousef, 2002). The OCSVM approach first maps training data into a high dimensional element territory (by means of a portion). Next, the algorithmic program iteratively finds the pinnacle edge hyperplane that best isolates the instructing data from the birthplace. The OCSVM might likewise be thoughtabout as a daily two-class SVM. Here the most classification involves all the coaching information, and therefore the second category is that the birthplace. on these lines, the hyperplane (or straight decision limit) compares to theThe accuracy rate of the planned system is ninety five. $72 \%$ and 
therefore the false positive rate is zero.7\%".

B. Classification Rule:

$\mathrm{f}(\mathrm{x})=\mathrm{hw}, \mathrm{xi}+\mathrm{b}$

Where $\mathrm{w}$ is that the customary vector and $\mathrm{b}$ could be a predisposition term. The OCSVM takes care of AN enhancement issue to discover the standard with pinnacle geometric edge. This grouping rule are utilized to dole out a name to a check model $\mathrm{x}$. In the event that $\mathrm{f}(\mathrm{x})<$ zero, we will in general mark $\mathrm{x}$ as AN abnormality, else, it is named conventional. All things considered, there's an exchange off between boosting the hole of the hyperplane from the root and consequently the assortment of instructing data focuses contained inside the locale isolated from the starting point by the hyperplane.

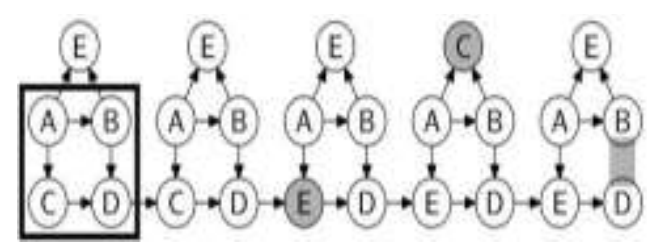

Figure.1. A graph with a normative substructure (boxed) and anomalies (shaded)

\section{Unsupervised Learning}

Calculation one makes use of three forms of diagram essentially based totally peculiarity detection(GBAD) (prepare dinner and Holder, 2007; Eberle and Holder, 2007; cook dinner and Holder, 2000; Yan and Han line, 2002) to deduce ability inconsistencies abuse every model. GBAD may be a diagram based way to address discovering peculiarities in statistics via coming across three elements: alterations, additions, and cancellations of vertices and edges. every specific trouble runs its very personal algorithmic program that finds a regulating substructure and makes an enterprise to discover the substructures that area unit comparative anyway not absolutely the image of the observed standardizing substructure. A regulating substructure could be a revenant subgraph of vertices and edges that, when amalgamated into one vertex, maximum packs the overall diagram. The rectangular shape in discern 1 recognizes A case of the regularizing substructure for the spoke to chart. Our execution makes use of SUBDUE (Ketkar et al., 2005) to discover regularizing substructures. The exceptional standardizing substructure might be described as a result of the only with

D. Borderline Description Length $(M D L): L(S, G)=$ $D L(G \mid S)+D L(S)(2)$

wherever $G$ is that the whole chart, $S$ is that the substructure being dissected, $\mathrm{DL}(\mathrm{G} \mid \mathrm{S})$ is that the depiction length of $\mathrm{G}$ once being compacted by $\mathrm{S}$, and $\mathrm{DL}(\mathrm{S})$ is that the portrayal length of the substructure being examined. Depiction length DL $(G)$ is that the base assortment of bits important to clarify chart $G$ (Eberle et al., 2011). Insider threats appear as meager extent differences from the regularizing substructures. This is because of business official threats choose to firmly impersonate real framework tasks aside from little varieties exemplified by ill-conceived conduct. we will in general apply 3 different methodologies for trademark such inconsistencies, referenced underneath.

\section{E. GBAD-MDL}

After locating the least complicated press standardizing substructure, GBAD-MDL scans for deviations from that regularizing substructure in resultant substructures. via breaking down substructures of a comparative length in mild of the reality that the standardizing one, reverence's inner the edges and vertices' names and closer to the course or endpoints of edges zone unit diagnosed. The leader abnormal of that place unit those substructures that the least changes area unit anticipated to give a substructure isomorphic to the regularizing one. In figure 4.1, the shaded vertex marked $\mathrm{E}$ is AN abnormality found via GBAD-MDL.

\section{F. $\quad G B A D-P$}

In qualification, GBAD-P scans for inclusions that, whenever erased, yield the regulating substructure. Inclusions made to a diagram zone unit saw as expansions of the regulating substructure. GBAD-P computes the opportunity of each expansion bolstered edge and vertex marks and along these lines abuses name data to get irregularities. The possibility is given by

$\mathrm{P}(\mathrm{A}=\mathrm{v})=\mathrm{P}(\mathrm{A}=\mathrm{v} \mid \mathrm{A}) \mathrm{P}(\mathrm{A})(3)$

Where $A$ represents a foothold or vertex attribute and $v$ represents its price. Chance $\mathrm{P}(\mathrm{A}=\mathrm{v} \mid \mathrm{A})$ may be generated by a Gaussian distribution

\section{G. GBAD-MPS}

At long last, GBAD-MPS considers erasures that, if reembedded, yield the regularizing substructure. To get these, GBAD-MPS looks at the parent structure. Changes in size and introduction inside the parent connote cancellations among the subgraphs. The premier anomalous substructures territory unit those with the smallest change value expected to make the parent substructures indistinguishable. In Figure four.1, the last substructure of A-B-C-D vertices is distinguished as anomalous by GBAD-MPS because of the missing edge among $\mathrm{B}$ and $\mathrm{D}$ set apart by the

\section{PROPOSAL WORK}

In the proposed methodology, the real goal is the mix of supervised and unsupervised learning.K-mean grouping technique and kNN arrangement strategy ought to give an answer for recognizing the atypical data. Data mining techniques is to distinguish the interruptions and for each methodology as various precision, false alert rate, and discovery rate. The accompanying figure demonstrates the portrayal of the proposed work 


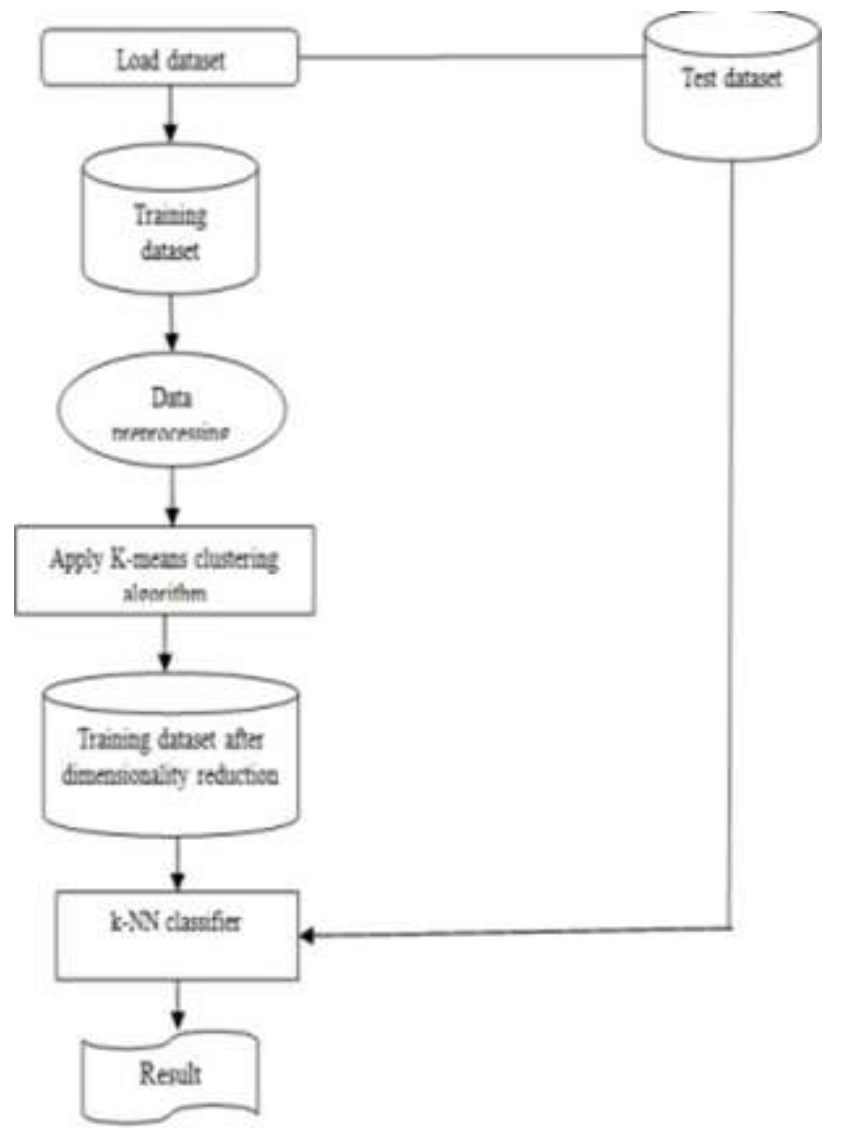

Figure 2: System Architecture

\section{CONCLUSION}

In this work, the fundamental commitment is in characterizing the groups and the closest neighbor by consolidating. "The measure planned is Euclidean separation dependent on the separation between information samples.K-implies approach is picked for grouping utilizing the separation measure to bunch both the preparation dataset. $\mathrm{k}-\mathrm{NN}$ in supervised learning based intrusion disclosure adequately. Here, k-NN maps the framework movement into predefined classes for example assault type or ordinary creates dependent on the preparation the marked dataset. k-NN based IDS, location rate and false caution rate. In this examination, we propose a $\mathrm{K}$-implies bunching approach and k-NN approach dependent on IDS that deal with the issue on the system. It makes IDS achieve high Detection Rate, False Alarm Rate, improve exactness and along these lines high interruption recognition capacity".

\section{REFERENCES}

1. Akiva, N. and M. Koppel (2012). Identifying distinct components of a multi-author document. In EISIC, pp. 205-209.

2. Al-Khateeb, T., M. M. Masud, L. Khan, and B. M Thuraisingham (2012). Cloud guided stream classification using class-based ensemble. In IEEE CLOUD, pp. 694-701.

3. Alipanah, N., P. Parveen, L. Khan, and B. M. Thuraisingham (2011). Ontology-driven query expansion using map/reduce framework to facilitate federated queries. In ICWS, pp. 712-713.

4. Alipanah, N., P. Parveen, S. Menezes, L. Khan, S. Seida, and B. M. Thuraisingham (2010). Ontology-driven query expansion methods to facilitate federated queries. In SOCA, pp. 1-8.

5. Brackney, R. C. and R. H. Anderson (Eds.) (2004, March). Understanding the Insider Threat. RAND Corporation. 